Published as: Auer, M. \& Griffiths, M.D. (2013). Voluntary limit setting and player choice in most intense online gamblers: An empirical study of gambling behaviour. Journal of Gambling Studies, 29, 647-660.

\begin{abstract}
Social responsibility in gambling has become a major issue for the gaming industry. The possibility for online gamblers to set voluntary time and money limits is a social responsibility practice that is now widespread among online gaming operators. The main issue concerns whether the voluntary setting of such limits has any positive impact on subsequent gambling behaviour and whether such measures are of help to problem gamblers. In this paper, this issue is examined through data collected from a representative random sample of 100,000 players who gambled on the win2day gambling website. When opening an account at the win2day site, there is a mandatory requirement for all players to set time and cash-in limits (that cannot exceed 800 Euros per week). During a three-month period, all voluntary time and/or money limit setting behaviour by a subsample of online gamblers $(n=5000)$ within this mandatory framework was tracked and recorded for subsequent data analysis. From the 5,000 gamblers, the 10\% most intense players (as measured by theoretical loss) were further investigated. Voluntary spending limits had the highest significant effect on subsequent monetary spending among casino and lottery gamblers.. Monetary spending among poker players significantly decreased after setting a voluntary time limit.. The highest significant decrease in playing duration was among poker players after setting a voluntary playing duration limit. The results of the study demonstrated that voluntary limit setting had a specific and significant effect on the studied gamblers. Therefore, voluntary limits appear to show voluntary limit setting had an appropriate effect in the desired target group (i.e., the most gaming intense players).
\end{abstract}

Key words: Online gambling; Responsible gambling; Social responsibility in gambling; Limit setting; Online lotteries; Online poker; Online casinos 


\section{Limit setting and player choice in most intense online gamblers: An empirical study of online gambling behaviour}

Social responsibility in gambling has become a major issue for the gaming industry (Griffiths, Wood, Parke \& Parke, 2007). To date there has been little research on the extent to which gaming companies are using social responsibility tools and engaging in socially responsible practices (Griffiths \& Wood, 2008). Social responsibility practices within the gambling industry typically involves policies, procedures and practices that promote of responsible gaming and minimise problem gambling (Griffiths \& Wood, 2008). A number of the social responsiility tools that have been incorporated by gaming companies have involved innovation in both information technology and technology more generally. In a recent study, Griffiths and Parke (2012) reported that regular gamblers endorse information technology developments as being helpful in reducing negative consequences associated with gambling.

One such social responsibility practice is the opportunity for players to pre-set limits for the amount of time and money they spend on gambling per day and/or per calendar month. This is a practice that is now widespread among online gaming operators (Wood \& Griffiths, 2010). Selflimiting options are viewed by some gaming companies and some researchers as a method of putting informed player choice into place at gaming sites (Griffiths \& Wood, 2008). Spending limit practices operated by current gaming operators come in a variety of forms. For instance, Wood and Griffiths (2010) reported that players' spending can be restricted in terms of deposit limits, play limits, loss limits, and bet limits. More specifically:

- Deposit limits - This refers to the maximum amount of money that a player can deposit into their play account at any given time. Winnings can either be included or excluded from this figure.

- Play limits - This refers to the maximum amount of money that a player can actually play with at any given time. As with deposit limits, winnings can either be included or excluded from this figure.

- Loss limits - This refers to the maximum amount of money that a player is allowed to lose at any one session.

- Bet limits - This refers to the maximum amount of money that can be bet on a single game, or on concurrent games. 
In addition to this, Wood and Griffiths (2010) also note that mandatory limits can either be fixed so that all games have the same limit, and/or all players have the same limit, or limits can be variable depending upon factors such as the type of game played, or the demonstrable wealth of the individual player. Furthermore, Wood and Griffiths (2010) have argued that fixed limits do not necessarily encourage and facilitate gamblers to take individual responsibility for managing and monitoring their own gambling expenditure.

Despite an increasing number of gaming operators utilizing social responsibility tools and practices, there is very little empirical research. Arguably the first empirical study was that of Smeaton and Griffiths (2004). They examined a wide range of socially responsibility practices by 30 British online gaming companies. There was a large range of bet limits across the gaming sites visited. The authors located only three sites that had no information about either minimum or maximum bet size. The minimum bet size found was $f 1$, whereas the maximum located (of the sites that set an upper limit) was $£ 20,000$. Many of the sites typically had $£ 10$ to $£ 25$ minimum bets, and $£^{250}$ to $£ 1000$ maximum bets. Smeaton and Griffiths argued that the larger the bet limit, the more chance that gamblers can run up debts that they cannot afford.

To date, and as far as the authors are aware, there is no empirical evidence to show that either higher mandatory spend limits or player self-set limits are associated with increased levels of problem gambling in either online or offline settings. Broda, LaPlante, Nelson, LaBrie, Bosworth and Shaffer (2008) examined the effects of player deposit limits on Internet sports betting by customers of bwin Interactive Entertainment. Their study examined 47,000 subscribers to bwin over a period of two years and compared the behaviour of players who tried to exceed their deposit limit with all other players. Deposit limit referred to the amount of money deposited into a player's spend account excluding any accumulated winnings. At the time of initial data collection in 2005, bwin set a mandatory deposit limit of no more than $€ 1000$ per day or $€ 5000$ per 30 days. Players could also set their own deposit limits (per 30 days) below the mandatory limits.

Overall, the study found that less than $1 \%$ of the players $(0.3 \%)$ attempted to exceed their deposit limit. However, Wood and Griffiths (2010) argued that the large mandatory limit may be the main reason for this finding as LaPlante at al (2008) noted that the majority of online gamblers never reached the maximum deposit limit. In fact, $95 \%$ of the players never deposited more than $€ 1050$ per 30 days (i.e., one-fifth of the $€ 5000$ maximum). Furthermore, LaPlante and colleagues 
did not distinguish between those who attempted to exceed either their own personally set deposit limits or mandatory limits. A Canadian study among Nova Scotian video lottery players found that responsible gaming $(R G)$ features (including player set spend limits) generally reduced the overall levels of player expenditure (Focal Research, 2007). However, as Wood and Griffiths (2010) note, the specific impact of the player set spend limit was not separated from the impact of the other RG features.

A study of 10,865 online gamblers from 96 different countries by the International Gaming Research Unit (2007) reported that over two-thirds of players (70\%) thought that voluntary spending limits would be a useful RG feature. Further focus group work from the same study found that the majority of players were opposed to mandatory spend limits. Mandatory spend limits were viewed by the focus groups as patronizing and overly restrictive. Bernhard, Lucas and Jang (2006) reported similar findings in their focus groups of Las Vegas gamblers. In this study, mandatory spend limits were strongly opposed, whereas player-set limits were more widely regarded as useful. However, problematic and pathological gamblers who are increasingly losing control of their time and money spending are not susceptible to voluntary responsible gaming features. This group of gamblers can effectively be protected by setting mandatory limits. Jurisdictions, like the one in Austria, often introduce these mandatory limits to protect the most vulnerable. The only way for the player to continue is to choose other gaming sites which do not protect players with mandatory limits. As appropriate prevention tools voluntary responsible gaming features require a certain level of self-awareness. Players should be introduced to responsible gaming from the very start of their gambling during registration on a specific site. Ideally they should be made familiar with videos, tutorials or other information material.

Griffiths, Wood and Parke (2009) carried out a study among Svenska Spel clientele examining players' attitudes and behaviour towards using social responsibility tools among 2,348 online gamblers (all clientele of Svenska Spel) who completed an online survey. The most useful feature was the setting of spending limits with over two-thirds of respondents $(70 \%)$ reporting the feature to be 'quite useful' or 'very useful'. Respondents were also asked which social responsibility features (if any) they had used. Over half (56\%) had used spending limits. Given the lack of empirical evidence on spending limits and whether they actually help in the prevention of problem gambling, the following study investigates the spending behaviour of online gamblers before and after they set themselves spend limits. 


\section{Method}

Participants: Data were collected from a representative random sample of 100,000 players who gambled on the win2day gambling website during a three-month test period. This sample comprised 5,000 registered gamblers who chose to set themselves limits while playing on win2day.

Gambling website description and procedure: The authors were given access to a large anonymized data set by a commercial gaming operator (win2day Entwicklungs- und Betriebsgesellschaft m.b.H), the online casino and lottery portal of Österreichische Lotterien GmbH and Casinos Austria AG. win2day has been online since 2003. win2day offers a wide range of lottery and casino games (as well as poker) to Austrian citizens. During the registration process, there is a mandatory requirement for all players to set time and cash-in limits. Furthermore, the weekly cash-in limit cannot exceed 800 Euros at any time during and after registration. Following registration, players can change the value of the weekly limit at any time (up to the mandatory 800 Euro per week limit). Limit increases only become effective after a 72-hour cooling off period. For instance, the player can limit the daily, weekly and/or monthly cash-in amount and the playing duration. The latter can be limited per playing session and/or per day. win2day protects its players by limiting the maximum cash-in amount per week at $€ 800$. Furthermore win2day offers additional responsible gaming features such as self-exclusion options (where players can temporarily or permanently self-exclude from gambling at win2day), educational content (e.g., video films including information on the nature of gambling and signs of problematic gambling), and a problem gambling diagnostic self-test (comprising questions similar to DSM-IV criteria). In the three-month test period, all voluntary limit setting behaviour by online gamblers was tracked and recorded for subsequent data analysis.

Monetary spending: Monetary spending was measured via theoretical loss. As shown in a recent study (i.e., Auer \& Griffiths, 2012), the theoretical loss is the most accurate and robust indicator of gambling intensity with regard to monetary involvement. The theoretical loss is computed as the product of bet size and house-advantage for each game being played. As Auer and Griffiths (2012) have demonstrated, the theoretical loss should always be used when gamblers with different gambling habits are being compared in terms of their involvement. The higher the theoretical loss, the higher the gambling involvement in terms of monetary spending. The computation of the theoretical loss as the product of bet size and house-advantage was applied to all games (e.g.,lottery games, casino games) with the exception of poker. Monetary ispending for poker was measured using the rake. The rake is a fixed percentage of the monetary bet that goes 
to the casino. In this study, poker refers to "social" poker in which gamblers compete with each other. This is clearly differentiated from 'video poker' which is a pure game of chance and thus a casino game. Previous studies have incorrectly tended to use bet size as a proxy measure of gambling involvement. As Auer and Griffiths have conclusively shown, bet size does not accurately take into account the house advantage of games and thus cannot be used to compare gamblers nor should it be ever used as a measure of individual gambling intensity. Auer and Griffiths also showed the same finding for when the number of games being played was used as a proxy measure of gaming intensity.

In this study, the effect of voluntary limit setting was calculated via the limit impact factor. To do this, the percentage change before and after the 30-day period after the limit was set was calculated. For the theoretical loss, the formula is as follows: impact factor $=$ (theoretical loss 30 days after divided by the theoretical loss 30 days before). An impact factor greater then ' 1 ' corresponds to a behavioural increase, and an impact factor less than ' 0 ' corresponds to a behavioural decrease. The effect if limit setting was studied by means of the $10 \%$ most intense players. This group of most intense players was identified by means of the behaviour (as measured by theoretical loss) in the 30 days before the limit was set. This approach to calculating 'gaming intensity' is a crucial factor when it came to the interpretation of limit setting effectiveness. Limits were deemed to have a significant effect if the mean theoretical loss or the mean playing duration after the limit setting event significantly decreased compared to before the limit setting event.

Data analysis: The data analysis was performed with the statistical package " $\mathrm{R}$ ". The analysis focused on the voluntary limit setting events following the registration process. Given the large number of statistical tests performed, significance levels were set at the $1 \%$ level. Mean changes in gambling behaviour before and after voluntary limit setting were performed via t-tests (by comparing the means of both theoretical loss and play duration before and after the limit setting events). Furthermore, changes in gambling behaviour were analysed overall and separately for casino, lottery and poker gambling. Only the 10\% most intense gamblers among each game type were taken into account. This subsegment of gamblers was chosen because they showed the highest losses based on their bet size and the types of games played (and therefore were arguably the ones who most need to set limits). A high theoretical loss can either occur through high bet sizes, through playing games with a high house advantage, or a combination of both. Lottery games have higher house advantages than casino games and thus lead to a higher theoretical loss. This might seem counter-intuitive as casino games are more problematic due to their event 
frequency. However, most of the time, the high theoretical loss of lottery games is compenstated by the low event frequency and low bet frequency that lottery gamblers usually show. Poker was taken into account via the rake, which as explained above is a fixed percentage of the stake that the player pays to the casino. A number of studies (e.g., Currie, Hodgins, Casey, et al. 2011) have shown that casino games correlate with increased harm. For that reason analysis was also performed separately for lottery, casino and poker games.

\section{Results}

\section{Findings relating to the total sample}

Effect of voluntary limit setting among the total sample: During the three-month test period, the sample of 5,000 gamblers produced a total of 22,002 limit setting acts (see Table 1). The mean number of limit setting acts per online gambler was $4.4(\mathrm{SD}=4.3)$. However, it should be noted that the changing of one limit can also result in the automatic changing of another. For instance, the monthly cash-in limit often determines a change in the weekly (as well as daily) cash-in limit. If the monthly cash-in limit is changed to $€ 800$, the weekly and daily cash-in limit cannot exceed that value. The same holds for the setting of play duration limits. Limiting the cash-in limit should consequently lead to a decreased playing behaviour either with respect to money spent or time spent.

\section{INSERT TABLE 1 ABOUT HERE}

In addition to theoretical loss, the following analysis also uses the playing duration as another measure of gambling intensity. In order to compute the playing duration for a certain time period, single playing sessions had to be identified. It was decided that the number of consecutive games belonged to one playing session if they were maximally 30 minutes apart. Therefore, a time gap of more than 30 minutes led to the recording of a new (and therefore separate) game session. The daily play duration corresponded to the sum of all sessions on that particular day. The playing duration for a specific time period corresponded to the sum of all daily playing durations for that time period.

\section{INSERT TABLE 2 ABOUT HERE}

Table 2 shows the limit impact factor with regard to theoretical loss in the group of most intense players and the five types of limit. Using t-tests, the results showed that the $10 \%$ most gaming intense players produced significantly less theoretical loss in the 30 days following voluntary limit 
setting (see Table 2). The 10\% most gaming intense players that chose a monthly cash-in limit produced $86 \%$ of the theoretical loss compared to 30 days before the limiting event. The setting of voluntary time limits also had a significant impact on spending behaviour than monetary limits although not as much as the setting of monetary limits (see Table 2). The 10\% most gaming intense players that chose a daily playing duration limit produced $90 \%$ of the theoretical loss compared to 30 days before voluntary limit setting.

\section{INSERT TABLE 3 ABOUT HERE}

Table 3 shows the impact of voluntary limit setting with regard to playing duration in the group of most intense players and the five types of limits. The results showed that the $10 \%$ most gaming intense players were playing significantly less often in the 30 days after setting voluntary time limits but only when choosing a daily duration limit. That is, in the 30 days after the limit setting event, the $10 \%$ most intense players that chose a daily playing duration limit spent $92 \%$ of the time playing compared to the 30 days before the limiting event. The setting of daily and weekly cash-in limits were almost significant at the $1 \%$ level in reducing play duration. Therefore, overall effect of setting voluntary limits on playing duration was less pronounced (and less significant) than the effect of voluntary limit setting on theoretical loss.

\section{Findings relating to the subsamples of lottery, casino and poker players}

The following analysis examined whether there were groups of players who differed with regard to the effect of limit setting. The data provided by win2day also contained data about which specific games the gamblers were playing. Therefore, subsequent analysis examined gamblers who played lottery, casino, and poker games. However, it must be noted the analysis were not mutually exclusive. Players who engaged in one type of gambling often engaged in other forms too.

Effect of voluntary limit setting among lottery players: Of the 5,000 gamblers who set limits within the three-month test period, 3,152 gamblers played lottery games (such as Lotto 6/45 and scratchcards). This sub-sample of 3,152 lottery gamblers carried out 12,222 acts of voluntary limit setting. The number and percentage of the various limits chosen is shown in Table 1. This shows that the distribution was very similar to the overall number and percentage of limits set in (see also Table 1). The number of limits per player was 3.9 ( $\mathrm{SD}=3.9)$. In order to show the impact of limit setting on time and money spent gambling, the $10 \%$ most intense gamblers were identified using the theoretical loss produced in lottery games in the 30 days before voluntary limit setting. 
Table 2 again shows that the theoretical loss significantly decreased among the top 10\% most gaming intense lottery players in the 30 days following all types of voluntary limit setting. The impact of the cash-in limits was higher than playing duration limits (Table 3).

Effect of voluntary limit setting among casino players: Out of the sample 5,000 gamblers who chose to set themselves limits in the three-month test period, 2,344 gamblers played casino games (such as slot games, video poker and cards). These 2,344 gamblers carried out 10,733 acts of voluntary limit setting. The number and percentage of the various limits chosen is shown in Table 1. This shows that the distribution was very similar to the overall number and percentage of limits (see also Table 1). The number of limits per player was $4.6(\mathrm{SD}=4.9)$. In order to show the impact of limit setting on time and money spent gambling, the 10\% most intense players were identified using the theoretical loss produced in casino games in the 30 days before voluntary limit setting. Table 2 shows that the theoretical loss, this time in casino games, significantly decreased in the top $10 \%$ of gaming intense players with respect to the theoretical loss in casino games in the 30 days prior to voluntary monetary limit setting (for daily, weekly and monthly cash-in limits). However, the setting of voluntary duration limits among casino players had no significant effect on theoretical loss after the limit setting event. Casino players showed a more significant decrease than the general population of gamblers. Casino gamblers who set themselves weekly cash-in limits spent $77 \%$ of the theoretical loss 30 days after voluntary limit setting compared to the theoretical loss 30 days prior. This is by far the most significant impact that limit setting had on gambling behaviour.

Table 3 shows that in general there was no significant decrease in play duration among casino players following voluntary limit setting (except for those who set monthly cash-in limits). The top $10 \%$ of most gaming intense casino players showed decreases in playing duration after voluntary limit setting that approached statistical significance for those who set voluntary daily and weekly cash-in but were non-significant for those who set session and daily duration limits.

Effect of voluntary limit setting among poker players: Out of the sample 5,000 gamblers who chose to set themselves limits in the thre-month test period, 759 gamblers played poker games (such as Texas Hold 'Em and Five Card Draw). These 759 gamblers carried out 2,893 acts of voluntary limit setting. The number and percentage of the various limits chosen is shown in Table 1 . The number of limits per person was $3.8(\mathrm{SD}=3.6)$. In order to show the impact of limit setting on time and money spent gambling, the $10 \%$ most intense poker players were created using the theoretical loss produced in poker games in the 30 days before voluntary limit setting. Table 2 
shows that poker rake decreased in the top $10 \%$ of gaming intense players in the 30 days following voluntary limit setting. However, this was only significant for those who set weekly monetary limits, and session and daily play duration limits (although the setting of daily and monthly monetary limits approached significance). Therefore, the decrease in rake for the top $10 \%$ of players was larger for time limits than for money limits. For instance, poker players who set themselves a daily playing duration limit only spent $73 \%$ of the rake they previously spent.

Table 3 shows that the setting of voluntary session and daily duration limits had a highly significant effect on overall play duration following the limit setting event. The voluntary setting of daily cash-in limits lowered play duration and approached statistical significance. The setting of weekly and monthly cash-in limits had no significant on poker play duration. Poker players who set themselves a daily playing duration limit only spent $70 \%$ of the time they used to spend playing poker. The analysis showed that intense poker gamblers changed their behaviour in a positive way after they set time rather than money limits.

Differences in limit setting between lottery, casino and poker players: The frequency of limit setting was different between the three types of player. Figure 1 show the $95 \%$ confidence intervals for the mean number of limit events per game type. Lottery $(\mathrm{M}=3.88$; $\mathrm{SD}=3.89 ; \mathrm{t}=-8.62, p<0.001)$ and poker players $(\mathrm{M}=3.81 ; \mathrm{SD}=3.36$; $\mathrm{t}=-9.71, \mathrm{p}<0.001)$ had a significantly lower number of limit events than the total population $(\mathrm{M}=4.40 ; \mathrm{SD}=4.29)$. Lottery $(\mathrm{t}=-6.93 ; p<0.001)$ and poker players $(\mathrm{t}=-7.58, \quad p<0.001)$ were also significantly different from casino players $(\mathrm{M}=4.58$; $\mathrm{SD}=4.90)$. Casino players tended to set more limits, but were not significantly different from the total population as the confidence intervals overlapped $(\mathrm{t}=1.76, p=0.78)$.

\section{INSERT FIGURE 1 ABOUT HERE}

\section{Discussion}

The results of this study clearly show that overall, voluntary limit setting had a specific and statistically significant effect on high intensity gamblers. Therefore, the study shows that voluntary limit setting had an appropriate effect in the desired target group (i.e., the most gaming intense players). More specifically, the analysis showed that (in general) gaming intense players specifically changed their behaviour in a positive way after they limited themselves with respect to both time and money spent. In most of the analyses (with the exception of poker players), the setting of voluntary time duration limits were less important than voluntary monetary limits. The 
results would seem to confirm the speculation made by Wood and Griffiths (2010) that voluntary time limits would be less effective than voluntary spending limits in changing gambling behaviour for the better among problem gamblers (assuming that the most gaming intense players in this study included problem gamblers).

Although the daily playing duration showed the highest impact on time spent gambling, there were no significant differences between voluntary cash-in and playing duration limits in overall time spent gambling following the limit setting event. The overall effect of limit setting on playing duration was much less than the overall effect on money spent. However, this might also be due to the fact that the distribution of monetary parameters was far more skewed and prone to outliers than the distribution of time parameters because the latter have natural lower and upper boundaries. For example the maximum daily time spend cannot exceed 24 hours.

It should also be noted that lottery games in general showed a very low frequency of play. Lotto is a very popular game but typically consists of players choosing the stake size and playing the game once or twice in a specified time period (e.g., once or twice a week). Therefore, there is a question as to whether setting limits (particularly time limits) would be of benefit as this study showed that limit setting by lottery players does not affect play duration. This would appear to be intuitively correct given that the structural characteristics (particularly event frequency) of biweekly lotteries would be unlikely to cause problems for players as compared to slot machines where event frequency can be very high (e.g., up to 30 times a minute), and which are known to have an association with problem gambling (e.g., Parke \& Griffiths, 2006; 2007, Meyer, Hayer \& Griffiths, 2009). That a limit on duration of session is irrelevant for playing the lottery has already been mentioned by Currie, Hodgins, Wang, el-Guebaly and Wynne, (2008). Given that the play duration for lottery games is typically much less than for casino or poker games, it could be argued that the setting of time limits is not needed for thr playing of discontinuous lottery games.

As outlined above, casino games (especially slot machine games) tend to have a very high event frequency and can be problematic for certain vulnerable groups of players (Meyer, et al, 2009). Given this association, it was pleasing that the results showed that voluntary monetary limit setting among players of these types of game showed highly significant decreases in the money lost as a direct result of voluntary limit setting. Among poker players, voluntary time limits showed a larger effect on the rake than monetary limits. Poker players were the only group where such an effect was observed. Given that playing poker is a more time intensive game than almost 
all other forms of gambling, it could be argued that voluntary limit setting impacting most on duration of play is a desirable outcome of limit setting in this particular type of player.

Overall - and excluding poker players - the analysis of the results shows that the setting of voluntary time limits are less important than the voluntary setting of monetary limits in significantly decreasing the theoretical losses among the most gaming intense players. The main concern of the analysis presented here is whether the playing behaviour of gamblers significantly changes after voluntary limits have been set. Here, the intensity of playing was measured in two ways (i.e., 'theoretical loss' and 'play duration'). The results do seem to provide evidence that voluntary limit setting has the desired effect in helping the most gaming intense players spend less time and/or money on their gambling. Given that the most gaming intense group of players set their spending limits below their actual theoretical loss, the results of this study clearly demonstrate that the most gaming intense players subsequently set voluntary limits appropriately and decreased their time and/or money playing the month after the limits were set.

There are, of course, many limitations with behavioural tracking data. As Auer and Griffiths (2011) have noted, behavioural tracking data (i) collects data from only one gambling site and says nothing about the person's online gambling in general (as online gamblers typically gamble on more than one site), (ii) always comes from unrepresentative samples (i.e., the players that use one particular internet gambling site), (iii) does not account for the fact that more than one person can use a particular account, and (iv) says nothing about why people gamble or why they engage in a particular online activity (such as limit setting). Another limitation is that once players reach their money or time limit, they may simply go and gamble on other online gambling websites.

Another more specific limitation in this study was whether the changes in observed behaviour were solely as a consequence of the voluntary limit setting.. Most players do not have limitless financial resources, therefore a high level of gambling cannot usually be sustained for very long. For this reason, high intensity gamblers' playing behaviour is likely to level off and/or decrease. Low intensity gamblers on the other hand are more likely to show an increase in their gambling rather than a decrease. In order to be able to make causal inferences, an experimental design would have to be constructed. Obviously this is not possible as limit setting is a voluntary event that cannot be enforced on players. However, if the results were purely a matter of chance, then differences between the types of limits and the types of gamblers would not likely have been observed. Many of the observed differences in this study were highly significant and showed that 
the setting of voluntary limits had a significant effect, and that different types of gamblers played differently as a result of voluntary limit setting.

Future analysis of data such as these could also include an examination of the players' behaviour when they get close to their time and money limits. It would be useful to know if they accelerate their behaviour (i.e., gamble more aggressively) or whether they reduce their level of gambling activity and become more passive. To be more specific, does the setting of limits create targets for gambling spend? Could the setting of limits be counterproductive (i.e., does the option to set limits actually encourage greater gambling)? This would help determine whether voluntary limits either encourage or inhibit gambling behaviour as the limit is reached. Such analysis might provide greater relevance to both public policy practitioners, and the gambling industry. Future studies should also investigate limit setting behaviour among less gambling intense players. It would be useful to know if this group of players knowingly set themselves limits that are higher than their actual gambling intensity. Among this group, the focus of limits would be to slow down the increase in gambling intensity rather than decrease the overall gambling intensity (as would be the aim among the most gaming intense players). The effect of limits can only be investigated by comparing gamblers who set themselves limits with similar gamblers who did not set themselves limits.

The focus of this study lies is on voluntary limit setting. The limited empirical evidence suggests that mandatory set limits are not liked by gamblers and that they prefer voluntary limits (e.g., International Gaming Research Unit, 2007). However, for voluntary limits to be effective in protecting players, a certain degree of readiness to change is required. The willingness and readiness to change is at the heart of the psychological 'stages of change' model (DiClemente \& Prochaska, 1992; Prochaska \& Prochaska, 1991). The 'stages of change' model assumes that there are varying levels of readiness for people to change their behaviour across five levels (i.e., precontemplation, contemplation, preparation, action, and maintenance). Furthermore, there is an oscillation between the different stages. Most vulnerable players who are unable to change may only have effective protection via mandatory limits.

It would appear from reviewing the small empirical literature base that there is evidence to suggest that most gamblers (irrespective of pathology) try to regulate their spending. Furthermore, it would appear from the data presented here that voluntary spend limits have the capacity to helpfully assist in that process. The evidence base suggests that the most appropriate responsible gambling strategy to be implemented by gaming companies would be for voluntary 
(rather than mandatory) pre-determined spending limits by players. This is because individuals are likely to vary widely in the amount of disposable income that they have available for leisure activities such as gambling. Players should therefore be required to set their own self-determined spending limits before they commence gambling. This is actually the case for the online platform win2day which provided the data for the analysis in this study. Such an action emphasizes individual responsibility for managing expenditure. It may also ensure that the player actively engages with at least one of the responsible gaming tools on offer. There would appear to be a consensus of expert opinion that encouraging player responsibility is a very effective long-term and preventive strategy for harm minimization. One concern regarding low-risk limits is that gamblers adhering to these limits may feel they are safe and impervious to harm. A related concern noted by Currie, Hodgins, Wang, el-Guebaly, Wynne and Miller, (2008) is that problem gamblers may justify continuing to gamble if they report staying within the limits.

Given that research in this important area is rather limited, it is recommended that the implementation and ongoing effectiveness of player set limits by gaming operators should be carefully monitored and evaluated. Follow-up research is needed to assess the impact of spending limits on player behaviour over time. Such research can provide a more accurate evaluation of the specific changes made, and can add valuable insight into the efficacy of such responsible gaming measures, contributing towards an exchange of best practice for both the national and international responsible gambling community. The term 'limit' appears to be unpopular, therefore the emphasis should instead be placed on offering game management tools that assist players in decisions about how much they want to spend gambling. Such management tools could also give players information about their actual gambling behavior and advice them based on their personal gambling patterns. Monaghan and Blaszczynski (2010) note that such systems should help players to reflect on the amount of time or money they have spent, compare expenditure to personally set limits, and consider whether they need appropriate self-regulatory action. Based on the findings of this study, it would appear that government policy makers, gaming regulators, and/or legislators should seriously consider making it mandatory for online gaming operators to introduce voluntary limit setting options for their players. This should include both time and money limit setting, particularly as time limit setting might be particularly good for some types of gamblers (i.e., poker players) whereas spending limits might be particularly helpful for other types (e.g., casino gamblers). 


\section{References}

Auer, M. \& Griffiths, M.D. (2012). Theoretical loss and gambling intensity: A simulation study. Gaming Law Review and Economics, 16, 269-273..

Bernhard, B.J., Lucas, A.F. \& Jang, D. (2006). Responsible gaming device research report. University of Nevada, Las Vegas International Gaming Institute.

Broda, A., LaPlante, D. A., Nelson, S. E., LaBrie, R. A., Bosworth, L. B. \& Shaffer, H. J. (2008). Virtual harm reduction efforts for Internet gambling: effects of deposit limits on actual Internet sports gambling behaviour. Harm Reduction Journal, 5, 27.

Currie, S.R., Hodgins, D.C., Casey, D.M., el-Guebaly, N., Smith, G.J, Williams, R.J., Schopflocher, D. P., Wood, R.T. (2011). Examining the predictive validity of low-risk gambling limits with longitudinal data. Addiction, 107, 400-406.

Currie, S.R., Hodgins, D.C., Wang, J., el-Guebaly, N., \& Wynne, H. (2008). In Pursuit of Empirically Based Responsible Gambling Limits. International Gambling Studies 8, 207-227.

Currie, S.R., D.C. Hodgins, D.C., Wang, J., el-Guebaly, N., Wynne, H. \& Miller, N.V. (2008b). Replication of low-risk gambling limits using Canadian provincial gambling prevalence data. Journal of Gambling Studies 24, 321-335.

DiClemente, C. C., Prochaska, J. O., Fairhurst, S. K., Velicer, W. F., Velasquez, M. M., \& Rossi, J. S. (1991). The process of smoking cessation: an analysis of precontemplation, contemplation, and preparation stages of change. Journal of Consulting and Clinical Psychology, 59, 295-304.

Focal Research Consultants (2007). Assessment of the behavioural Impact of the responsible Gaming Device (RGD) Features: Analysis of Nova Scotia Player-Card Data. The Windsor Trial. Report prepared for the Nova Scotia Gaming Corporation.

Griffiths, M.D. \& Auer, M. (2011). Approaches to understanding online versus offline gaming impacts. Casino and Gaming International, 7(3), 45-48. 
Griffiths, M.D. \& Wood, R.T.A. (2008). Responsible gaming and best practice: How can academics help? Casino and Gaming International, 4(1), 107-112.

Griffiths, M.D., Wood, R.T.A. \& Parke, J. (2009). Social responsibility tools in online gambling: A survey of attitudes and behaviour among Internet gamblers. CyberPsychology and Behavior, 12, 413-421.

Griffiths, M.D., Wood, R.T.A., Parke, J. \& Parke, A. (2007). Gaming research and best practice: Gaming industry, social responsibility and academia. Casino and Gaming International, 3(3), 97-103.

International Gaming Research Unit (2007). The global online gambling report: An exploratory investigation into the attitudes and behaviours of internet casino and poker players. eCOGRA (e-Commerce and Online Gaming Regulation and Assurance).

Meyer, G., Hayer, T. \& Griffiths, M.D. (2009). Problem Gaming in Europe: Challenges, Prevention, and Interventions. New York: Springer.

Monaghan, SM \& Blaszczynski, A. (2010) Electronic gaming machine warning messag-es: information versus self-evaluation. Journal of Psychology: Interdisciplinary and Applied, 144, 83-96.

Parke, J. \& Griffiths, M.D. (2006). The psychology of the fruit machine: The role of structural characteristics (revisited). International Journal of Mental Health and Addiction, 4, 151-179.

Parke, J. \& Griffiths, M.D. (2007). The role of structural characteristics in gambling. In G. Smith, D. Hodgins \& R. Williams (Eds.), Research and Measurement Issues in Gambling Studies (pp.211-243). New York: Elsevier.

Parke, A. \& Griffiths, M.D. (2012). Beyond illusion of control: An interpretative phenomenological analysis of gambling in the context of information technology. Addiction Research and Theory, 20, 250-260.

Prochaska, J.O.P., \& Prochaska, J.M. (1991). Why don't people change? Why don't continents move? Journal of Pyschotherapy Integration, 9, 83-102. 
Smeaton, M. \& Griffiths, M.D. (2004). Internet gambling and social responsibility: An exploratory study, CyberPsychology and Behavior, 7, 49-57.

Wood, R.T.A. \& Griffiths, M.D. (2010). Social responsibility in online gambling: Voluntary limit setting. World Online Gambling Law Report, 9(11), 10-11. 
Table 1: Types of limit and percentage of occurrence among online gamblers

Limit type

All online players $(n=5000)$

Daily cash-in limit

Weekly cash-in limit

Monthly cash-in limit

Session playing duration limit

Daily playing duration limit

Total

Online lottery players $(n=3152)$

Daily cash-in limit

Weekly cash-in limit

Monthly cash-in limit

Session playing duration limit

Daily playing duration limit

Total

Online casino players $(n=2,334)$

Daily cash-in limit

2,439

Weekly cash-in limit

2,981

3,718

10,733

Total

Online poker players $(n=759)$

Daily cash-in limit

Monthly cash-in limit

Session playing duration limit

Daily playing duration limit

Total
$23 \%$

773

822

3,063

$25 \%$

$29 \%$

$32 \%$

$7 \%$

$7 \%$

$100 \%$

$28 \%$

$35 \%$

$7 \%$

$8 \%$

$100 \%$
$30 \%$

$32 \%$

$188 \quad 6 \%$

189

$7 \%$

2,893

$100 \%$ 
Table 2: Impact of limit setting on theoretical loss (€) after limit setting among most intense online gamblers (Limit Impact Factor) comparing play 30 days before and 30 days after limit setting event)

Cash-in Limit

\section{Daily Weekly Monthly Session Daily}

All players $(n=5000)$

Mean

(SD)

t value

(p)

Lottery players $(n=3,152)$

Mean

(SD)

t value

$(p)$

Casino players $(n=2,344)$

Mean

(SD)

t value

(p)

Poker players $(n=759)$

Mean

(SD)

$\mathrm{t}$ value

(p)
0.87

$(0.7)$

$-4.15$

$(<0.001)$
0.87

(0.63)

(0.6)

$-3.55$

$(<0.001)$
0.86

0.91

0.9

$(0.7)$

(0.7)

(0.7)

$-4.47$

$-4.47$

$-2.88$

$-3.19$

$(<0.0001)$

$(<0.0001)$

$(<0.01)$ 
Table 3: Impact of limit setting on Playing Duration after limit setting among most intense online gamblers (Limit Impact Factor) comparing play 30 days before and 30 days after limit setting event)

Cash-in Limit

Daily Weekly Monthly Session Daily

All players $(n=5000)$

Mean

$(\mathrm{SD})$

t value

$(p)$

Lottery players

$(n=3,152)$

Mean

(SD)

0.91

(0.8)

0.91

(0.8)

0.88

(0.8)

0.96

0.99

0.92

(0.6) (0.6)

$-2.24$

(0.013)

$-0.89$

$-1.86$

(0.03)

Casino players

$(n=2,344)$

Mean

(SD)

$\mathrm{t}$ value

$(p)$

Poker players $(n=759)$

Mean

(SD)

$\mathrm{t}$ value

$(p)$
0.91

(0.8)

$-1.72$

(0.04)

0.93

(0.4)

$-1.52$

(0.07)

(0.19)
0.88

$-0.8$

$-2.29$

(0.01)

0.97

(0.6)

$-0.43$

(0.33)
$-0.28$

(0.39)

Duration Limit

Daily 
Figure 1: Mean number of limit events per game type

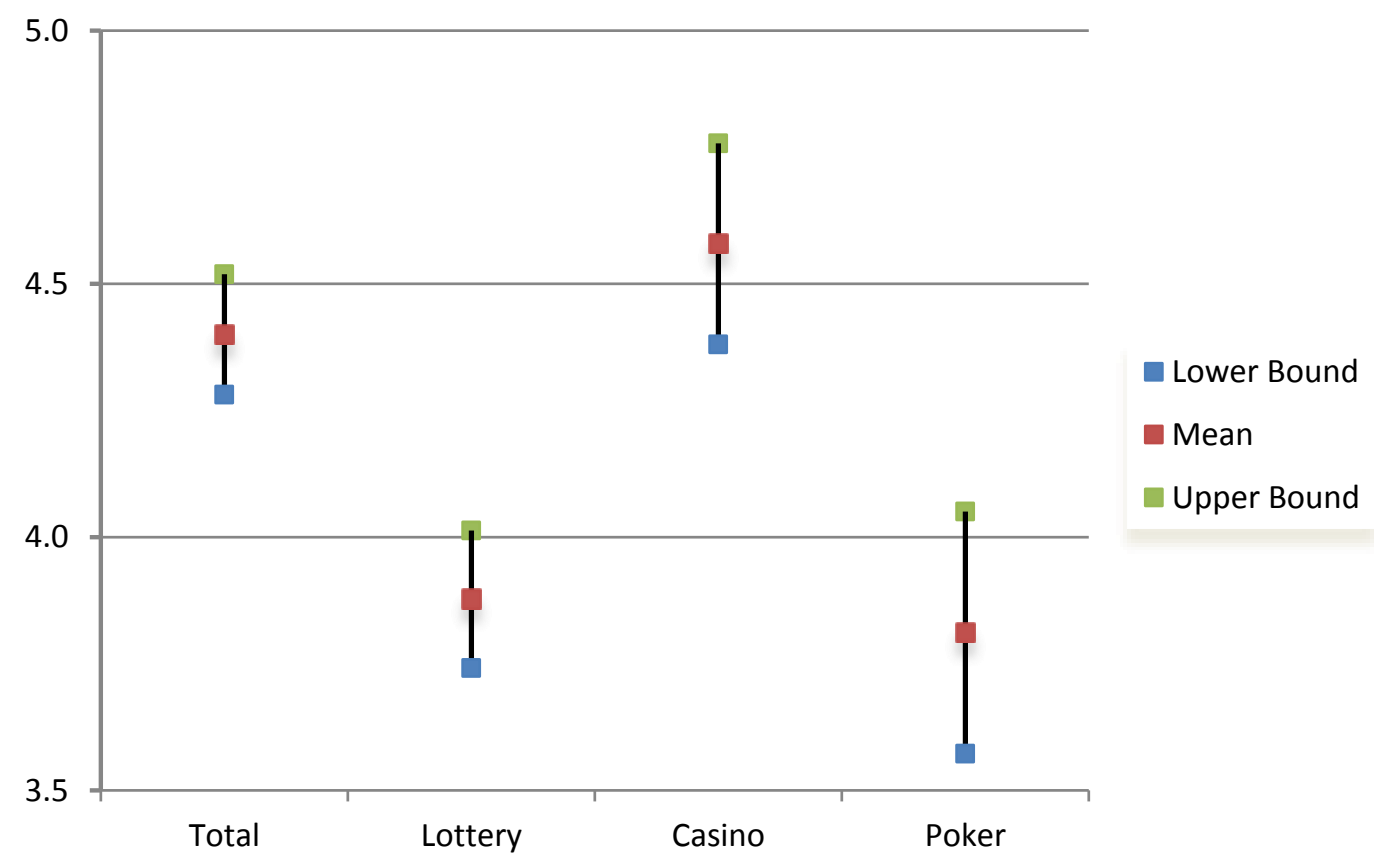

